\section{Ferritin expression in the periodontal tissues of primates}

\author{
Wenxue Huang, ${ }^{1}$ Wei Li, ${ }^{2}$ \\ Weidong Zhu, ${ }^{1}$ Juan Liu, ${ }^{3}$ Jianxia Hou, ${ }^{1}$ \\ Huanxin Meng ${ }^{1}$ \\ ${ }^{1}$ Department of Periodontology, Peking \\ University School and Hospital of \\ Stomatology; National Clinical Research \\ Center for Oral Diseases; National \\ Engineering Laboratory for Digital and \\ Material Technology of Stomatology; \\ Beijing Key Laboratory of Digital \\ Stomatology, Beijing \\ ${ }^{2}$ Department of Periodontology, \\ The Affiliated Hospital of Qingdao \\ University; Key Laboratory of Oral \\ Clinical Medicine, College of \\ Stomatology, Qingdao University \\ ${ }^{3}$ Department of Stomatology, Beijing \\ Friendship Hospital, Capital Medical \\ University, Beijing, China
}

\begin{abstract}
Ferritin, an iron-binding protein, is composed of two subunits, ferritin heavy chain and ferritin light chain. It regulates many biological functions, such as proliferation, angiogenesis, and immunosuppression. The objective of this study was to determine the expression and distribution of ferritin in the periodontal tissues of primates. First, we assessed the expression of ferritin in primary cultured cells isolated from human periodontal tissues using the polymerase chain reaction and immunofluorescent staining in vitro. Second, we investigated the expression and distribution of ferritin in the periodontal tissues of Macaca fascicularis, human gingival tissues, and human gingival carcinoma tissues using immunohistochemistry. Both protein and mRNA of ferritin were constitutively present in human primary cultured cells, including those from the dental apical papilla, periodontal ligament, dental pulp, and gingival epithelium, as well as gingival fibroblasts. In $M$. fascicularis tissues, the immunohistochemical staining was particularly strong in blood vessel and mineralizing areas of the dental pulp and periodontal ligament. Ferritin heavy chain exhibited specific immunopositivity in the stratum basal of the epithelium in human gingival tissue, and strong immunostaining was found in peripheral regions of gingival carcinoma sites. Ferritin is constitutively pres-
\end{abstract}

ent and widely distributed in the periodontal tissues of primates. Ferritin may play roles in epithelial proliferation, vascular angiogenesis, and mineralization in these tissues.

\section{Introduction}

Iron is essential for biological processes such as cell proliferation, energy production, oxygen transport, electron transport, and DNA synthesis. ${ }^{1}$ Ferritin is major cellular iron-storage protein, composed of ferritin light chain (FTL) and ferritin heavy chain (FTH). As a component of regulatory network that maintains iron homeostasis, ferritin releases iron via ferritinophagy and sequesters excess iron to avoid damage by reactive oxygen species (ROS). ${ }^{2}$ The FTH and FTL subunits are encoded by different genes and have distinct functions: the former has ferroxidase activity and converts ferrous ions to ferric ions, ${ }^{3}$ while the latter has many carboxy groups which act as iron nucleation sites. ${ }^{4}$

Ferritin also regulates biological functions independent of iron, such as cell proliferation, ${ }^{5}$ angiogenesis, ${ }^{6}$ the epithelial-tomesenchymal transition, ${ }^{7}$ and the induction of chemokine transduction signaling pathways. ${ }^{8}$ Furthermore, new research has demonstrated that ferritin interacts with other proteins, such as $\mathrm{P}^{9} 3^{9}$ and nuclear receptor coactivator $4,{ }^{10}$ influencing their functions and altering cellular responses. The functions of ferritin have not been thoroughly clarified.

Oral cavity is a bacteria-rich environment, and since iron is a key element for the growth, survival, and cellular processes of most bacteria, ${ }^{11}$ host and pathogen may compete for it. Ferritin expression and secretion increase during infection, hypoxia, and inflammation, ${ }^{12}$ and may act against pathogens by sequestering iron and stimulating the host immune response. Previously, we demonstrated that ferritin was higher expressed in human periodontal ligament (PDL) tissues than in the other human tissues examined, including heart, skin, kidney, bone marrow, spleen, testis, lung, liver, skeletal muscle, brain, thymus. ${ }^{13}$ In human oral tissues, different mesenchymal stem cells (MSCs) are obtained to treat bone defects, spinal cord injury, and inflammatory diseases. ${ }^{14-17}$ However, the expression, distribution, and biological functions of ferritin in periodontal tissues have few reports. Human oral-derived cells are easily harvested. ${ }^{18} \mathrm{We}$ assessed the expression of ferritin in primary cultured cells isolated from human periodontal tissues using the polymerase chain reaction (PCR) and immuno-
Correspondence: Prof. Jianxia Hou, Department of Periodontology, Peking University School and Hospital of Stomatology, 22 Zhongguancun South Avenue, Haidian District, Beijing 100081, China. Tel. +86.10.82195367.

E-mail: jxhou@163.com

Prof. Huanxin Meng, Department of Periodontology, Peking University School and Hospital of Stomatology, 22 Zhongguancun South Avenue, Haidian District, Beijing 100081, China. Tel. +86.10.82195374.

E-mail:kqhxmeng@bjmu.edu.cn

Key words: Ferritin; ferritin heavy chain; Macaca fascicularis; periodontal ligament; dental pulp; gingiva; carcinoma of gingiva.

Funding: This work was supported by grants from the National Natural Science Foundation of China (No. 81470738) to JH.

Contributions: WH, contribution to design experiments and data acquisition, manuscript drafting; WL, WZ, JL, contribution to study design and data acquisition; JH, HM, contribution to study design, manuscript critical revision. All authors read and approved the final manuscript.

Conflict of interest: The authors report no conflicts of interest related to this study.

Received for publication: 23 May 2019. Accepted for publication: 13 August 2019.

This work is licensed under a Creative Commons Attribution-NonCommercial 4.0 International License (CC BY-NC 4.0).

(C) Copyright: the Author(s), 2019

Licensee PAGEPress, Italy

European Journal of Histochemistry 2019; 63:3046 doi:10.4081/ejh.2019.3046

fluorescent staining in vitro firstly. The anatomy and physiology of $M$. fascicularis are similar to humans. Gingiva is a part of periodontal tissues and could be obtained from patients of health and gingival carcinoma. We used immunohistochemical assays to detect the location of ferritin in periodontal tissues of $M$. fascicularis, human gingival tissues, and human gingival carcinoma tissues in vivo. To date, no studies have been carried out to reveal the localization and expression of ferritin in periodontal tissues of primates. This study demonstrated that ferritin was constitutively and widely expressed in the cells of periodontal tissues which played roles in proliferation, vascular angiogenesis, inflammation, and mineralization. Ferritin may be a new biomarker and therapeutic target in the pathogenesis and treatment of periodontitis and oral cancer. 


\section{Materials and Methods}

\section{Ethics statement}

This research protocol was approved by the Review Board and the Ethics Committee of Peking University School and Hospital of stomatology (PKUSSIRB2011007), and was approved by the Experimental Animal Welfare Ethics section of the Peking University Biomedical Ethics Committee (Protocol LA2008-006). All clinical samples were acquired from patients who received treatment in the Peking University Hospital of Stomatology, agreed to the use of their specimens for research purposes, and gave written informed consent.

\section{Cell culture}

Human dental apical papilla cells, PDL cells, human gingival fibroblasts, human dental pulp cells, and human gingival epithelial cells were isolated and passaged according to the methods previously described. ${ }^{19-21}$ Briefly, the apical papilla, PDL, and pulp cells were obtained from wisdom teeth extracted from young healthy donors who had no dental or periodontal diseases. Gingival epithelial cells and fibroblasts were isolated from healthy volunteers who received crown-lengthening surgery. The samples of dental pulp and gingival connective tissue were relatively large and contained abundant cells, so the success rates of pulp cells and gingival fibroblasts were $100 \%$ in the absence of microbial contamination. Dental apical papillae had to be obtained from relatively young volunteers. The proliferation capacity of gingival epithelial cells was the poorest among the cell types. PDL tissue from one tooth was the smallest sample, so the success rate of PDL cells (PDLCs) was the lowest at $20 \%$.

Apical papilla, PDL, pulp, and gingival fibroblasts were maintained in Dulbecco's modified Eagle's medium (DMEM; Gibco, Grand Island, NY, USA) containing 10\% $(\mathrm{v} / \mathrm{v})$ fetal bovine serum (FBS), 1\% (v/v) antibiotics (penicillin $\mathrm{G}$ and streptomycin; Gibco). Gingival epithelial cells were maintained in keratinocyte serum-free medium (ScienCell, San Diego, CA, USA). All cells were cultured in a humidified atmosphere of $5 \% \mathrm{CO}_{2}$ at $37^{\circ} \mathrm{C}$. After $12 \mathrm{~h}$ of incubation, cells in 12-well culture plates (Corning Inc., Corning, NY, USA) were attached to the wall, and the morphology of cells were fully extended. These cells were used for protein detection. We cultured cells in 6well plates for mRNA examination when the cells reached $80 \%$ confluence after 24 $48 \mathrm{~h}$ of incubation. Cultured cells were used between the second and fourth passages.

\section{PCR assessment of ferritin mRNA expression in primary cells}

Total RNA was extracted from the above primary cultured cells using TRIzol ${ }^{\circledR}$ Reagent (Invitrogen, Carlsbad, CA, USA), then used for cDNA synthesis with a Reverse Transcription System (Toyobo, Osaka, Japan). RNA quality was assessed as the absorbance ratios at $260 \mathrm{~nm} / 230 \mathrm{~nm}$ and $260 \mathrm{~nm} / 280 \mathrm{~nm}$. The synthetic cDNA was mixed with gene-specific primers and Taq Master Mix (Solarbio Science \& Technology Co., Beijing, China), then the PCR was carried out. The gene-specific primers used for PCR were as follows: FTH, forward: CAGGTGCGCCAGAACTACCA, reverse: CCACATCATCGCGGTCAAAG; FTL, forward: ACCATGAGCT CCCAGATTCGTC, reverse: ACACATC
ATCGCGGTCGAAATAG; D-glyceraldehyde-3-phosphate dehydrogenase (GAPDH), forward: ATGGGGAAGGTGAAGGTCG, reverse: GGGGTCATTGATGGCAACAAT. All experiments were carried out in duplicate.

\section{Immunofluorescence assessment of ferritin protein expression in primary cells}

The expression of the FTH and FTL proteins was assessed by immunofluorescence. The primary cultured cells were seeded on glass coverslips. After $12 \mathrm{~h}$ of culture, they were fixed in $4 \%$ paraformaldehyde at room temperature for $10 \mathrm{~min}$. Then, endogenous peroxidase was blocked by $3 \% \mathrm{H}_{2} \mathrm{O}_{2}$ at room temperature for $10 \mathrm{~min}$. To block nonspecific reactions, cells were treated with $10 \%$ normal rabbit serum at room temperature for $30 \mathrm{~min}$. The cells were then incubated with goat antihuman primary antibodies against FTH and FTL (Santa Cruz Biotechnology Inc., Santa Cruz, CA, USA) at $4^{\circ} \mathrm{C}$ overnight. After the cells were washed three times with phosphate-buffered saline (PBS), they were incubated with rabbit anti-goat secondary antibody (Zhongshan Golden Bridge Biotechnology, Beijing, China) for $1 \mathrm{~h}$ at room temperature. Nuclei were counterstained with 4',6-diamidino-2-phenylindole (DAPI). Lastly, the coverslips were mounted on glass slides and the cells were viewed under a confocal Zeiss Axiovert 650 microscope at $488 \mathrm{~nm}$ (green, FTL), $510 \mathrm{~nm}$ (red, FTH), and $405 \mathrm{~nm}$ (blue, DAPI). Cellular images were captured on an LSM 5 Exciter confocal imaging system (Carl Zeiss, Oberkochen, Germany). As negative controls, normal goat IgG replaced the primary antibodies.
A

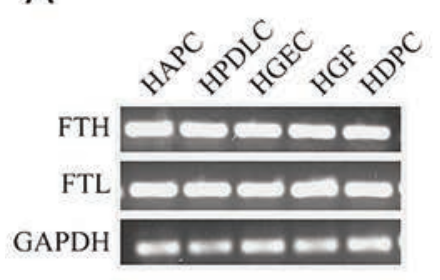

B

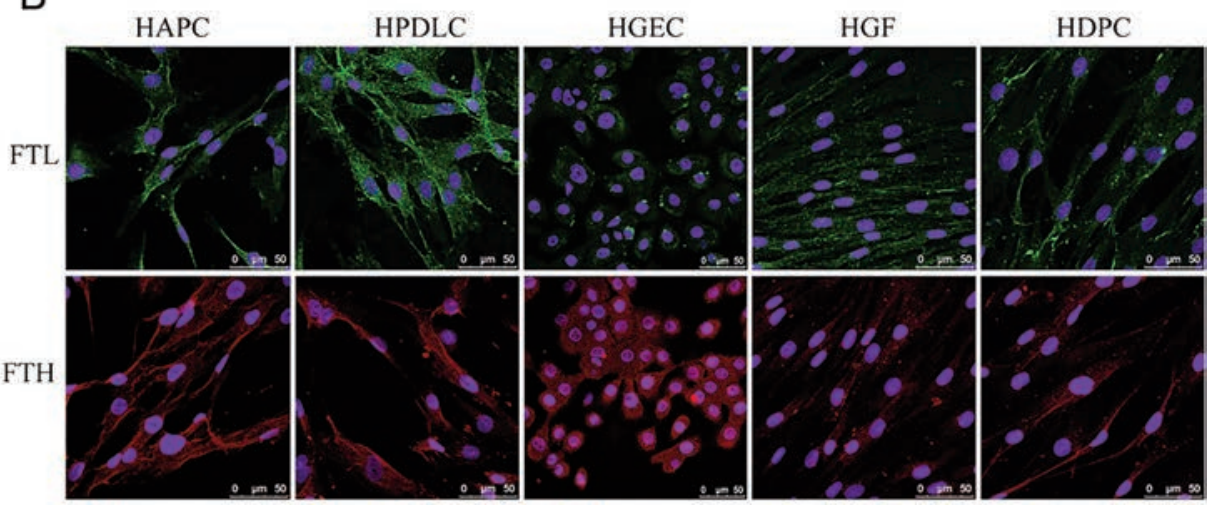

Figure 1. Expression of FTH and FTL in human primary cells isolated from periodontal tissues. A) mRNA expression of FTH and FTL in primary cultured apical papilla cells (HAPC) (lane 1), periodontal ligament cells (HPDLC) (lane 2), gingival epithelial cells (HGEC) (lane 3), gingival fibroblasts (HGF) (lane 4), and pulp cells (HDPC) (lane 5); GAPDH served as the internal control. B) Representative images of protein expression of FTH (red), FTL (green), and cell nucleus (blue) in primary cultured cells. Scale bar: $50 \mu \mathrm{m}$. 


\section{Study population}

Twelve healthy participants and 12 patients with carcinoma of the gingiva were recruited from Peking University Hospital of Stomatology, China. The healthy individuals, who received crown-lengthening surgery in the Department of Periodontology, were examined to determine their clinical periodontal status. Periodontal examination included probing depth ( $\mathrm{PD} \leq 3 \mathrm{~mm}$ ) using a William's periodontal probe at six sites of each tooth, and bleeding index (BI $\leq 20 \%$ ) after probing on two sides per tooth as pre- viously described. ${ }^{22}$ Gingival carcinoma samples were collected from patients who had been diagnosed in the Department of Oral and Maxillofacial Surgery. The samples were fixed in $4 \%$ paraformaldehyde and stained with hematoxylin and eosin (H\&E).

\section{Experimental animals}

Three adult $M$. fascicularis (long-tailed macaque) males were used in this study (Laboratory Animal Center of Academy of Military Medical Sciences), and had been used in earlier research in our laboratory. ${ }^{23-26}$ In brief, the monkeys were 5.5-6.0 years old and weighed $5.1-5.5 \mathrm{~kg}$. After euthanasia with an overdose of ketamine hydrochloride, the mandibular premolars and molars were dissected. Alveolar bone surrounding the teeth was included. These specimens were fixed in $10 \%$ paraformaldehyde, demineralized in buffered $10 \%$ EDTA at $37^{\circ} \mathrm{C}$, dehydrated, and embedded in paraffin. Mesic-distal sections parallel to the dental long axis were cut at $5 \mu \mathrm{m}$ on a microtome. Some sections were stained with H\&E.
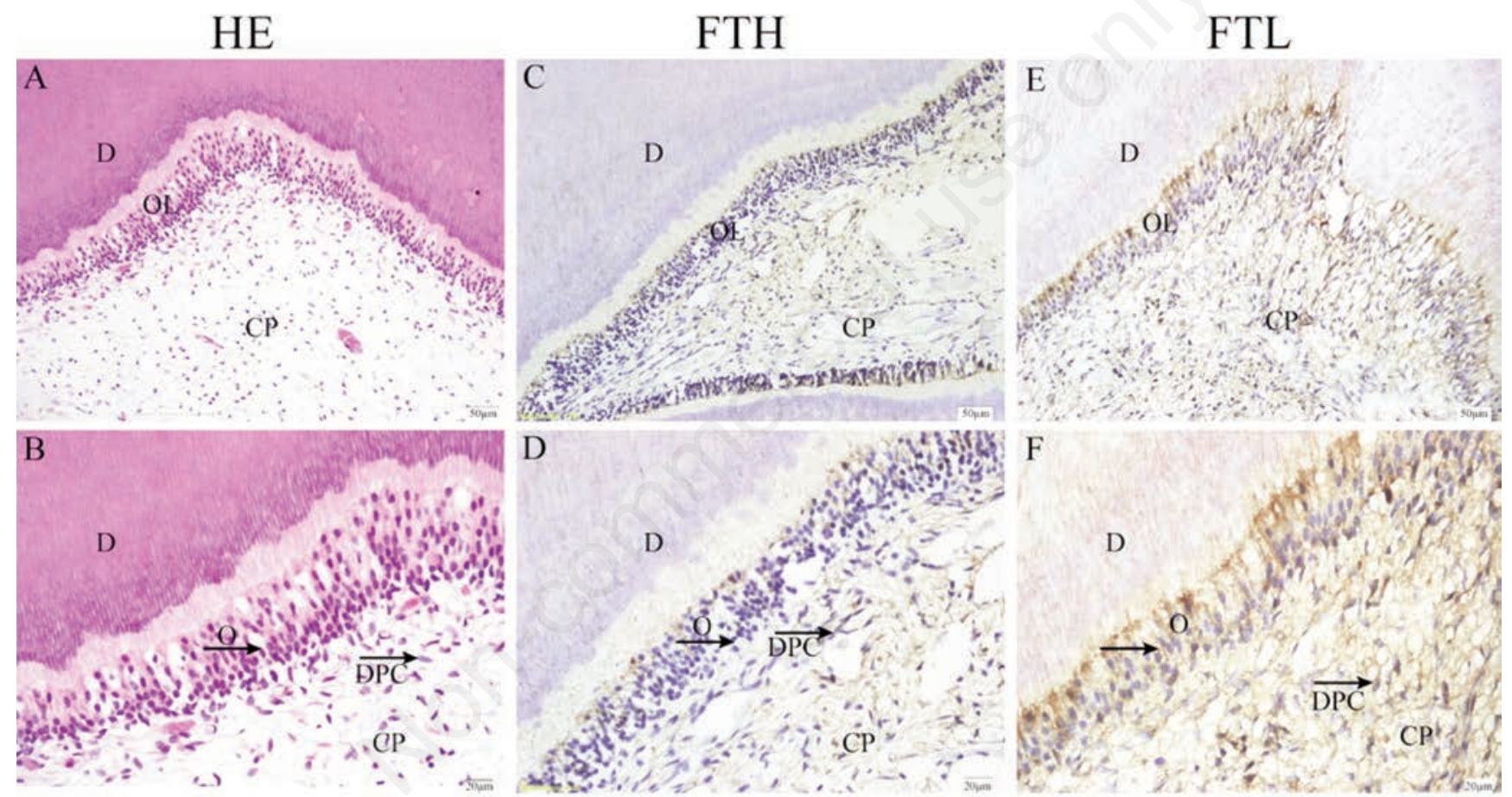

\section{IRS (Mean \pm SD) Odontoblastic layer}

FTH

FTL
$10.71 \pm 2.268$

$9.53 \pm 2.939$
$2.79 \pm 1.369$

$7.76 \pm 1.393$
$p$-Value $<0.001$

$p$-Value 0.032

Figure 2. Immunohistochemical localization of FTH and FTL in dental pulp from $M$. fascicularis. Representative sections from specimens of dental pulp. A,B) Hematoxylin-eosin staining. C,D) Immunostaining of FTH in the odontoblastic layer and pulp proper. E,F) Positive expression of FTL in the pulp core. Data are presented as mean \pm SD. IRS, immunoreactive score; FTH, ferritin heavy chain; FTL, ferritin light chain; D, dentin; CP, central pulp; O, odontoblast; DPC, dental pulp cell. Scale bars: A,C,E) $50 \mu \mathrm{m} ; \mathrm{B}, \mathrm{D}, \mathrm{F}) 20 \mu \mathrm{m}$. 


\section{Immunohistochemical staining of ferritin and statistical analysis}

The paraffin-embedded sections were deparaffinized with xylene and rehydrated in a series of ethanol. Endogenous peroxidase was inactivated by incubation with $3 \%$ $\mathrm{H}_{2} \mathrm{O}_{2}$ at room temperature for $10 \mathrm{~min}$. Antigen was retrieved by trypsin digestion at $37^{\circ} \mathrm{C}$ for $10 \mathrm{~min}$. Then the specimens were rinsed three times in PBS and treated with $10 \%$ normal goat serum at room temperature for 30 min followed by incubation with rabbit antihuman primary antibodies against FTH and FTL (Santa Cruz
Biotechnology, Inc.) overnight at $4^{\circ} \mathrm{C}$. After washing three times with PBS for 5 min each, the expression and distribution of FTH and FTL were visualized using an immunohistochemistry kit and a DAB detection kit (Zhongshan Golden Bridge Biotechnology, Beijing, China). After counterstaining with hematoxylin, the sections were mounted. Images were captured on a light microscope with a digital camera (BX51/DP72, Olympus, Tokyo, Japan).

Immunoreactive score (IRS) with modification was used to evaluate immunohistochemical staining of ferritin in the odonto- blast, PDLCs adjacent to the alveolar bone and cementum. ${ }^{27}$ Briefly, IRS = staining intensity (SI) x percentage of positive cells (PP). SI was defined as: 0, negative; 1 , weak; 2 , moderate; 3 , strong. PP was determined as: 0 , negative; $1,0-10 \%$ positive cells; $2,10 \%$ $50 \%$ positive cells; $3,50 \%-75 \%$ positive cells; and $4,75 \%-100 \%$ positive cells. Ten visual fields of each specimen were chosen for the IRS evaluation. Statistical analyses were performed using SPSS Statistics 20.0 software and carried out using $t$-test for testing two groups. The value of $\mathrm{P}<0.05$ was considered statistically significant.
HE
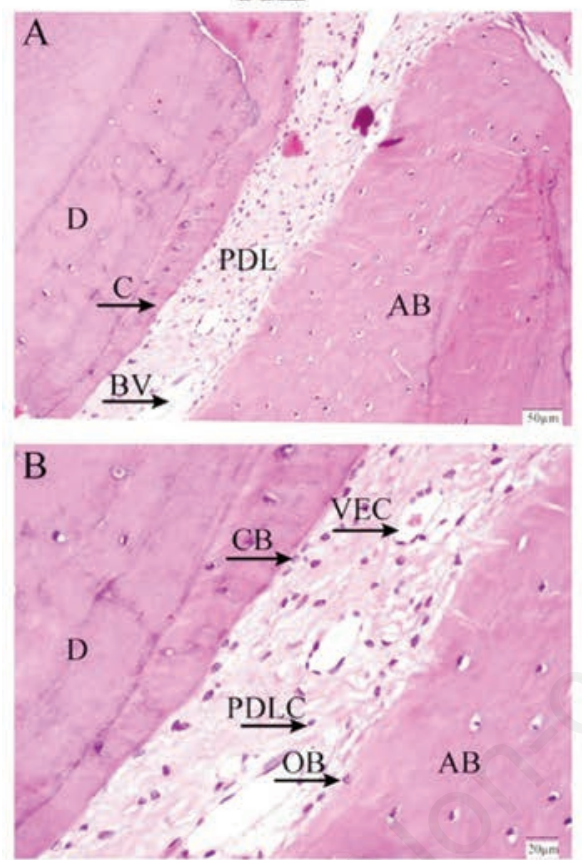

FTH

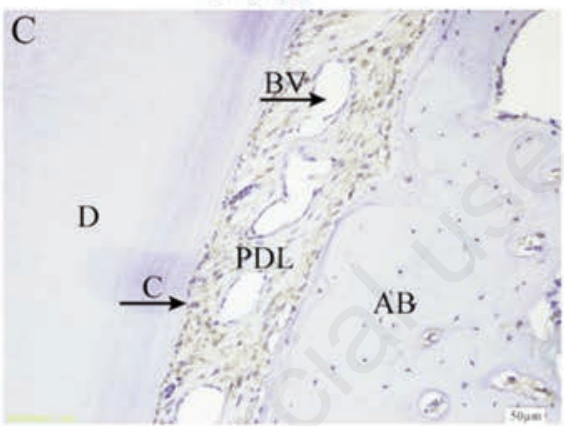

D

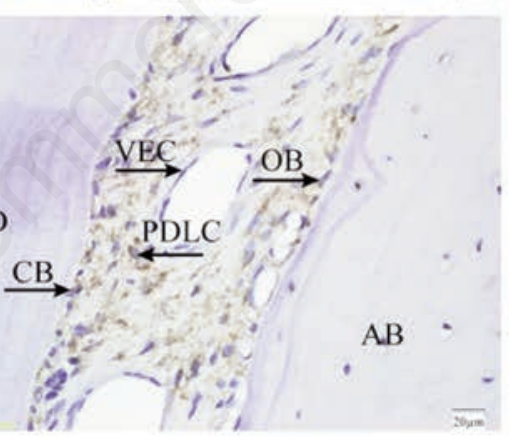

FTL
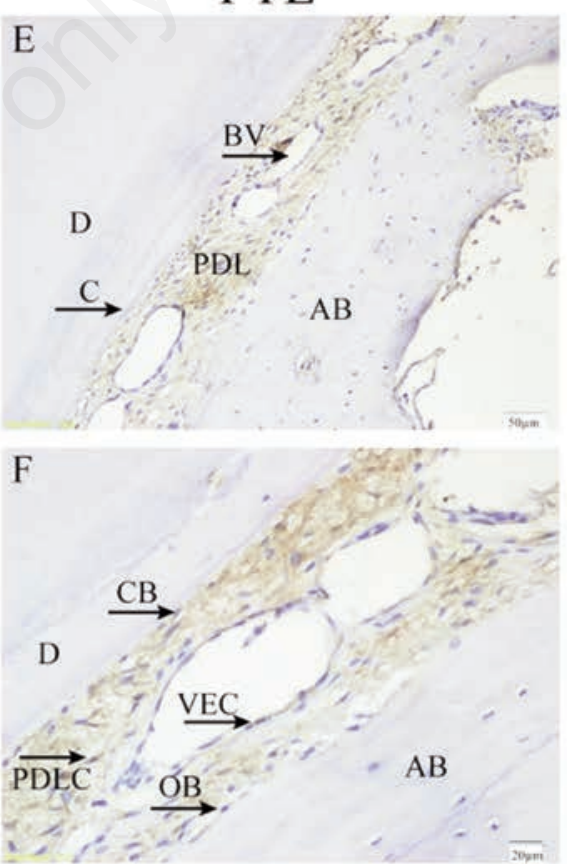

IRS $($ Mean \pm SD $)$
PDLCs adjacent to

\author{
cementum
}

FTH

FTL
$5.44 \pm 1.617$

$6.29 \pm 1.993$
PDLCs adjacent to

alveolar bone

$6.11 \pm 1.811$

p-Value 0.252

$6.82 \pm 2.243$

p-Value 0.472

Figure 3. Immunohistochemical localization of FTH and FTL in periodontal ligament from $M$. fascicularis. A,B) Hematoxylin-eosin staining. C,D) Strong FTH expression in cementoblasts, osteooblasts, and adjacent periodontal ligament cells. E,F) FTL expression in periodontal ligament cells and vascular endothelial cells. Data are presented as mean \pm SD. IRS, immunoreactive score; FTH, ferritin heavy chain; FTL, ferritin light chain; D, dentin; $C$, cementum; PDL, periodontal ligament; $A B$, alveolar bone; $B V$, blood vessel; $C B$, cementoblast; $O B$, osteoblast; PDLC, periodontal ligament cell; VEC, vascular endothelial cell. Scale bars: A,C,E) $50 \mu \mathrm{m}$; B,D,F) $20 \mu \mathrm{m}$. 


\section{Results}

Ferritin expression was constitutive in human primary cells isolated from periodontal tissues in vitro

The total RNA from apical papilla, PDL, pulp, and gingival epithelial cells and fibroblasts was analyzed by RT-PCR. The results revealed that FTH and FTL mRNA transcripts were strongly expressed in all these cell-types (Figure 1A). Cell immunofluorescence assays of FTH and FTL protein confirmed the PCR results (Figure 1B). The immunostaining of FTH and FTL was mainly localized in the cytoplasm. Ferritin was most abundant in apical papilla, PDL, and gingival epithelial cells followed by gingival fibroblasts and pulp cells.

\section{Ferritin was widely distributed in periodontal tissues of M. fascicularis}

Although we had shown that ferritin was present in these cells, its distribution in periodontal tissues needed to be systematically examined, because cells in different sites perform different functions. Completely healthy human periodontal tissues are difficult to obtain, while nonhuman primates are appropriate experimental models for research, because their anato- my and physiology are similar to humans. Microscopic analysis of periodontal tissues showed that specimens reacted to anti-FTH and anti-FTL antibodies. In detail, dental pulp, the PDL, alveolar bone, and bone marrow were positively stained for FTH and FTL.

We used immunohistochemical assays to determine the distribution of FTH and FTL in the dental pulp of $M$. fascicularis. The results showed that FTH staining was concentrated in the odontoblastic layer lining the outermost layer of dental pulp, and the staining of FTH in the pulp core was weaker than odontoblastic layer (Figure 2D). Meanwhile, both pulp core and odontoblastic layer were positive for FTL (Figure 2F). In the PDL, the immunoreactivity of FTH was more positive in the PDLCs adjacent to alveolar bone and cementum (Figure 3D), while FTL expression was positive in all PDLCs and VECs (vascular endothelial cells) of the PDL (Figure 3F). In the root furcation, both FTH and FTL expression were present in the osteocytes of alveolar bone (Figure 4 D,F). In the bone marrow, adipocytes and fibroblasts lining the marrow center and osteoblasts lining the marrow cavity showed strong positive staining for FTH and FTL (Figure 5 D,F).
Characteristic ferritin heavy chain expression in healthy human gingiva and gingival carcinoma tissues

We investigated ferritin expression in gingival tissues, which were not available for $M$. fascicularis. In gingival connective tissue, FTH was positive in the VECs (Figure 6). In the oral epithelium, FTH was positive in the stratum basal. The basal cells of oral epithelium are stem cells; they are immature and undifferentiated, with a marked capacity for division and proliferation. Therefore, we examined the expression of FTH in gingival carcinomas. Immunohistochemical staining showed the expression of ferritin in the peripheral areas of tumors and areas of inflammatory cell infiltration was stronger. Cellular FTH may affect the proliferation and invasion of cancer cells.

\section{Discussion}

To the best of our knowledge, this is the first study to reveal the localization and expression of ferritin in the periodontal tissues of primates. Investigation of human primary cultured cells provided evidence that ferritin is constitutively expressed in human apical papilla cells, PDL cells, gingival fibrocytes, dental pulp cells, and gingi-
HE
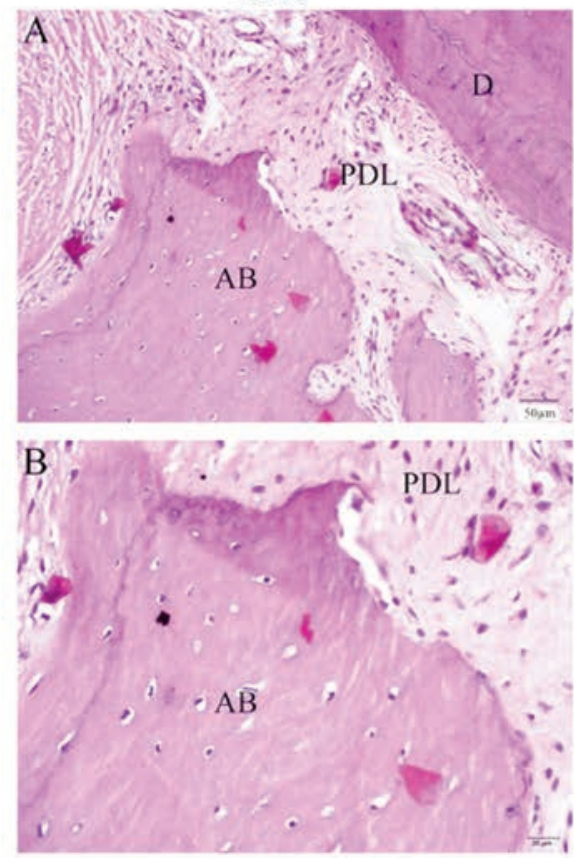

FTH

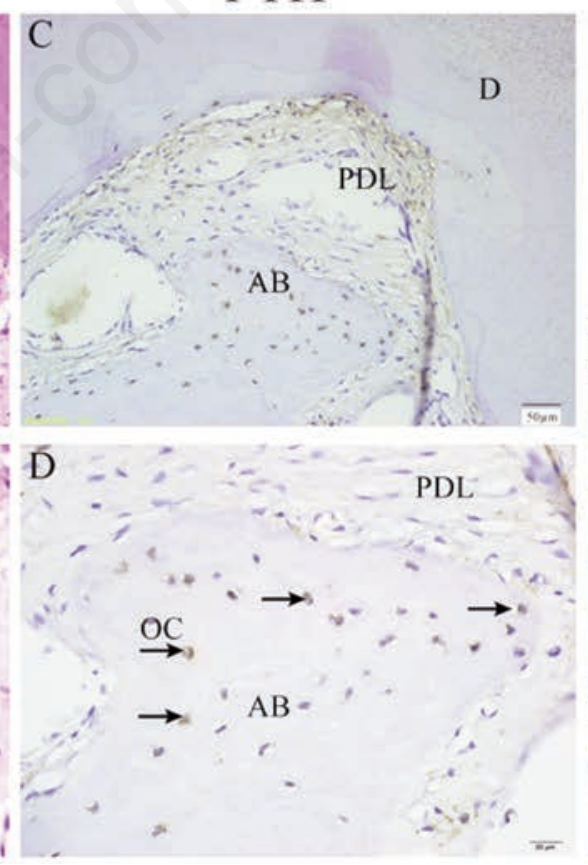

FTL

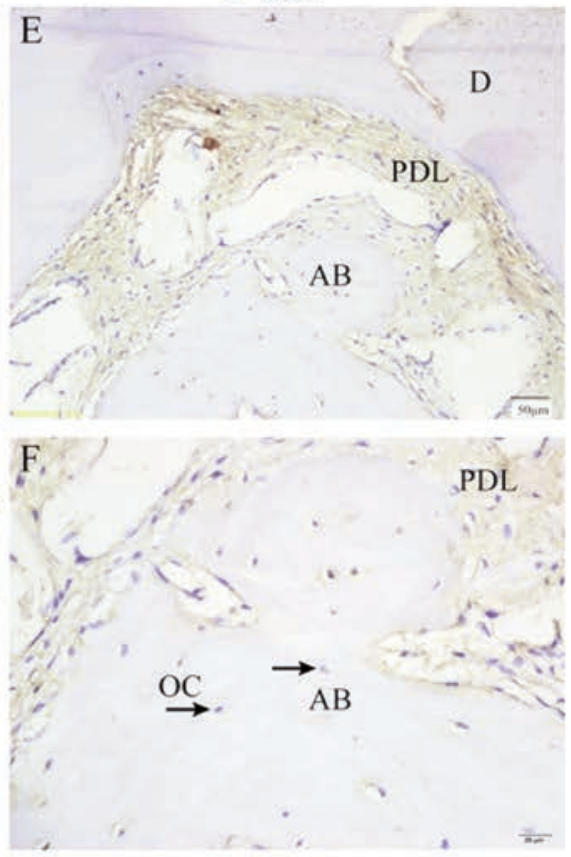

Figure 4. FTH and FTL expression pattern in tooth furcation of $M$. fascicularis. A,B) Hematoxylin-eosin staining. C,D,E,F) Both FTH and FTL expression were present in the osteocytes of alveolar bone. D, dentin; PDL, periodontal ligament; AB, alveolar bone; OC, osteocyte. Scale bars: A,C,E) $50 \mu \mathrm{m}$; B,D,F) $20 \mu \mathrm{m}$. 
val endothelial cells. Dental pulp, gingiva, and PDL are all vascularized connective tissues. After injury and inflammation, their healing potential is very beneficial. In part, this healing potential is associated with local cells which secrete factors that promote cells differentiation and neovascularization. Previous research has shown that ferritin has regulatory effects on angiogene- sis. ${ }^{28}$ The expression of FTL in vascular endothelial cells in connective tissues deserves further investigation to determine whether it has a proangiogenic effect in the periodontal tissues. The local, abundantly expressed ferritin might contribute to healing. The ratio of FTH and FTL varies depending on tissue type and could be modified by pathological conditions. ${ }^{5}$ According to the immunohistochemical results, PDLCs and pulp cells expressed high levels of ferritin. Moreover, these cells which have mineralization activity like osteoblasts contain both FTH and FTL. ${ }^{29}$ This demonstrates that both FTH and FTL may be involved in the process of mineralization. Recently, it has been reported that ferritin, especially FTH, negatively modulates the
HE
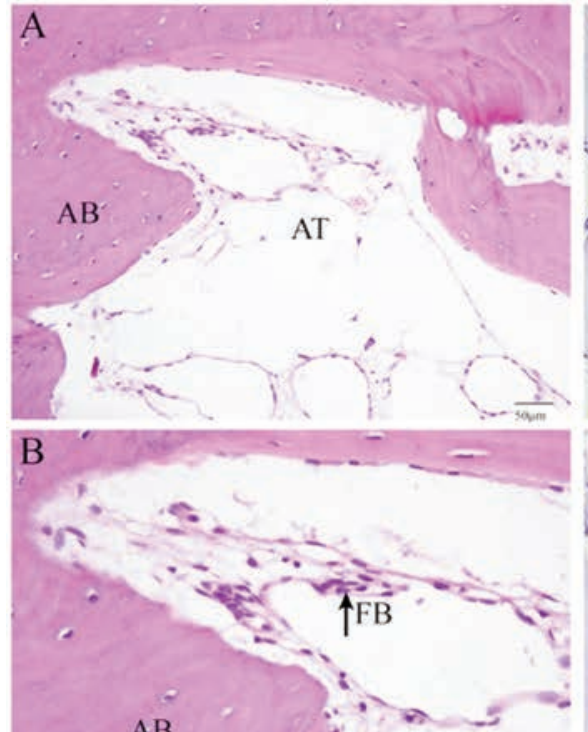

$\mathrm{AB}$

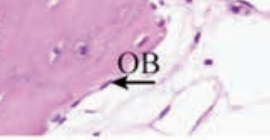

FTH

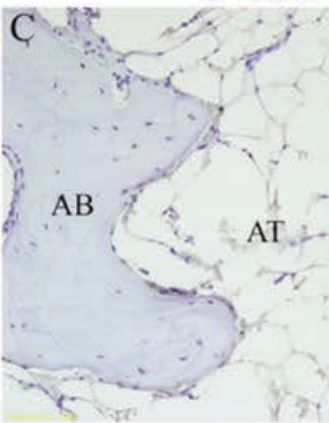

D

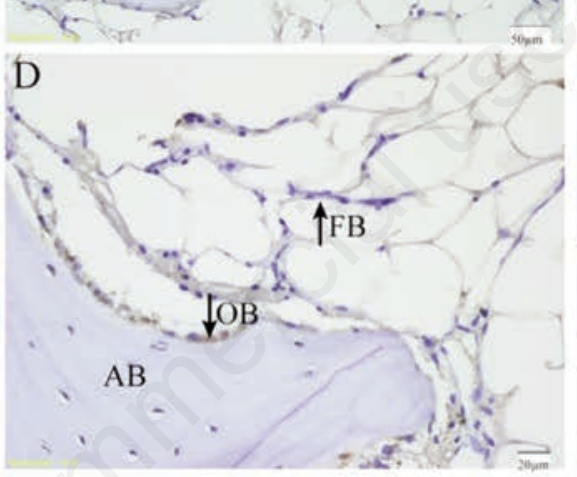

FTL

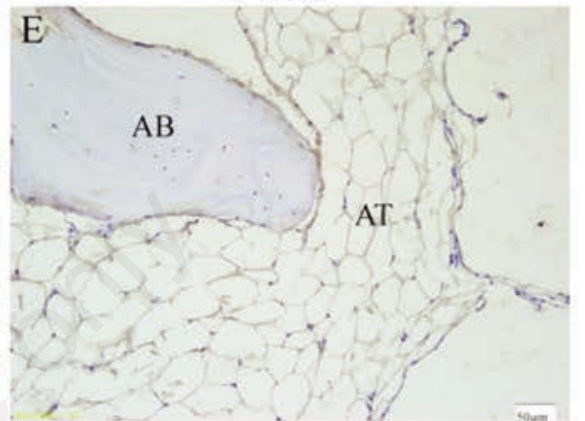

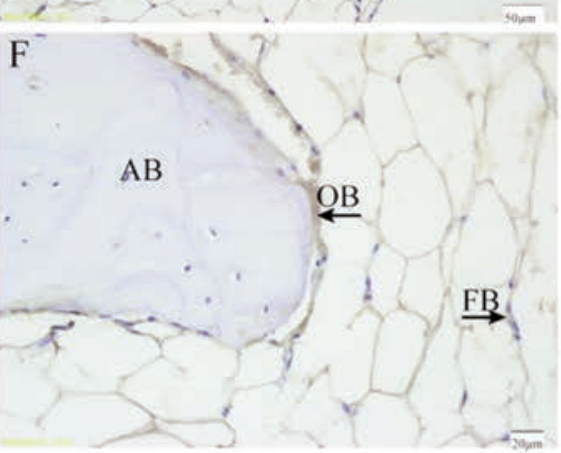

Figure 5. FTH and FTL expression pattern in bone marrow between roots of teeth from $M$. fascicularis. A,B) Hematoxylin-eosin staining. C-F) Adipocytes, fibroblasts, and osteoblasts show strong positive staining for FTH and FTL. FTH and FTL immunostaining in similar areas. AB, alveolar bone; AT, adipose tissue; FB, fibroblast; OB, osteoblast. Scale bars: A,C,E) 50 m; B,D,F) $20 \mu \mathrm{m}$.

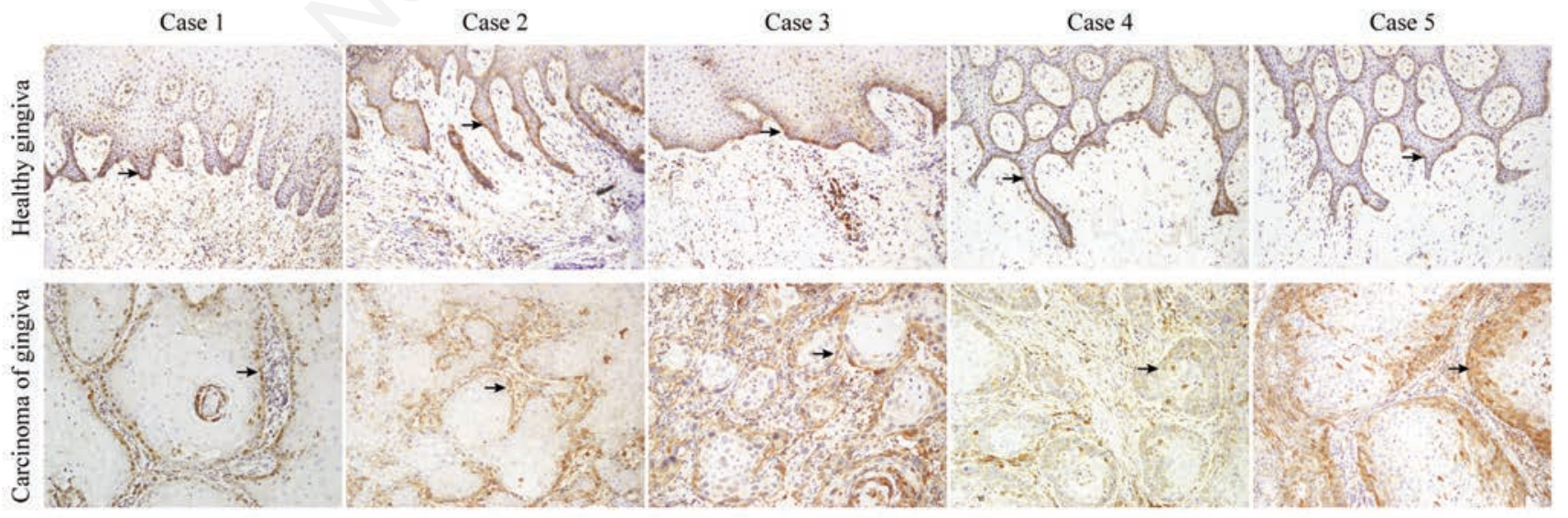

Figure 6. Expression of FTH in human gingival samples and gingival carcinoma tissue. Immunohistochemical analysis of the location of FTH expression in human healthy gingival tissues and gingival carcinoma tissues. As indicated by the arrows, we found strong immunoexpression of FTH in basal cells of the epithelium in healthy gingiva. At the same time, peripheral regions of tumor islands and areas of inflammatory cell infiltration in tumorous connective tissues also expressed abundant FTH. Original magnification: 200x. 
differentiation of osteosarcoma cells and human smooth muscle cells into osteoblasts via ferroxidase activity. ${ }^{30-32}$ We deduce that an autocrine or paracrine ferritin loop might be formed in the local environment to promote mineralization homeostasis in healthy tissues and enhance osteogenesis in disease sites. Further studies are needed to uncover the roles of ferritin in cytodifferentiation and mineralization.

Ferritin is strongly associated with cancer. As previous reports, serum ferritin is elevated in patients with multiple malignancies, including neuroblastoma, ${ }^{33}$ melanoma, ${ }^{34}$ renal cell carcinoma, nonsmall-cell lung cancer. ${ }^{35}$ The level of serum ferritin is higher, the disease is more progressive, and the survival is shorter. Serum ferritin in cancer patients consists mainly of FTH and is a potential biomarker. ${ }^{36}$ However, the sources and functions of ferritin in serum are unclear. Moreover, ferritin inhibits immune responses, increases angiogenesis, and promotes proliferation. Evidence has demonstrated that extracellular ferritin has immunosuppressive effects on myeloid cells and lymphocytes..$^{37,38}$ In a mouse tumor model, the addition of ferritin increases intratumor blood vessel density significantly. ${ }^{39}$ Targeting FTH and FTL with shRNA results in growth reduction of cancer stem cells. ${ }^{40}$

In this study, we found that FTH expression was positive in the stratum basal of oral epithelium and blood capillaries, was enhanced in the periphery of tumor sites where proliferation ability is high, and was upregulated in tumorous connective tissues of inflammatory cell infiltration. FTH is a candidate marker of stem cells in gingival carcinoma and may be a source of elevated serum ferritin through secreting by tumor cells. Ferritin may be involved in the pathogenesis and development of gingival carcinoma by regulating cells proliferation, angiogenesis and immune response. The phenomenon and mechanism need further confirmation and investigation.

In conclusion, we identified that ferritin was constitutively present and widely distributed in the periodontal tissues of primates and might play roles in epithelial proliferation, vascular angiogenesis, inflammation and mineralization in these tissues.

\section{References}

1. Walter PB, Knutson MD, PalerMartinez A, Lee S, Xu Y, Viteri FE, et al. Iron deficiency and iron excess damage mitochondria and mitochondrial DNA in rats. Proc Natl Acad Sci USA 2002;99:2264-9.

2. Dowdle WE, Nyfeler B, Nagel J, Elling
RA, Liu S, Triantafellow E, et al. Selective VPS34 inhibitor blocks autophagy and uncovers a role for NCOA4 in ferritin degradation and iron homeostasis in vivo. Nat Cell Biol 2014;16:1069-79.

3. Boyd D, Vecoli C, Belcher DM, Jain SK, Drysdale JW. Structural and functional relationships of human ferritin $\mathrm{H}$ and L chains deduced from cDNA clones. J Biol Chem 1985;260:1175561.

4. Wade VJ, Levi S, Arosio P, Treffry A, Harrison PM, Mann S. Influence of sitedirected modifications on the formation of iron cores in ferritin. $\mathrm{J}$ Mol Biol 1991;22:1443-52.

5. Torti FM. Regulation of ferritin genes and protein. Blood 2002;99:3505-16.

6. Kitagawa T, Kosuge H, Uchida M, Dua MM, Iida Y, Dalman RL, et al. RGDconjugated human ferritin nanoparticles for imaging vascular inflammation and angiogenesis in experimental carotid and aortic disease. Mol Imaging Biol 2012;14:315-24.

7. Aversa I, Zolea F, Ierano C, Bulotta S, Trotta AM, Faniello MC, et al. Epithelial-to-mesenchymal transition in FHC-silenced cells: the role of CXCR4/CXCL12 axis. J Exp Clin Cancer Res 2017;36:104.

8. Li R, Luo C, Mines M, Zhang J, Fan GH. Chemokine CXCL12 Induces binding of ferritin heavy chain to the chemokine receptor CXCR4, alters CXCR4 signaling, and induces phosphorylation and nuclear translocation of ferritin heavy chain. J Biol Chem 2006;281(:37616-27.

9. Biamonte F, Battaglia AM, Zolea F, Oliveira DM, Aversa I, Santamaria G, et al. Ferritin heavy subunit enhances apoptosis of non-small cell lung cancer cells through modulation of miR125b/p53 axis. Cell Death Dis 2018;9: 1174.

10. Santana-Codina N, Mancias JD. The role of NCOA4-mediated ferritinophagy in health and disease. Pharmaceuticals (Basel) 2018;11. pii: E114.

11. Wandersman C, Delepelaire P. Bacterial iron sources: from siderophores to hemophores. Annu Rev Microbiol 2004;58:611-47.

12. Alkhateeb AA, Connor JR. The significance of ferritin in cancer: anti-oxidation, inflammation and tumorigenesis. Biochim Biophys Acta 2013;1836:24554.

13. Hou J, Yamada S, Kajikawa T, Ozaki N, Awata T, Yamaba S, et al. Role of ferritin in the cytodifferentiation of periodontal ligament cells. Biochem
Biophys Res Commun 2012;426:643-8.

14. Mammana S, Gugliandolo A, Cavalli E, Diomede F, Iori R, Zappacosta R, et al. Human gingival mesenchymal stem cells pretreated with vesicular moringin nanostructures as a new therapeutic approach in a mouse model of spinal cord injury. J Tissue Eng Regen Med 2019;13:1109-21.

15. Pizzicannella J, Gugliandolo A, Orsini T, Fontana A, Ventrella A, Mazzon E, et al. Engineered extracellular vesicles from human periodontal-ligament stem cells increase VEGF/VEGFR2 expression during bone regeneration. Front Physiol 2019;10:512.

16. Cianci E, Recchiuti A, Trubiani O, Diomede F, Marchisio M, Miscia S, et al. Human periodontal stem cells release specialized proresolving mediators and carry immunomodulatory and prohealing properties regulated by lipoxins. Stem Cells Transl Med 2016;5:20-32.

17. Trubiani O, Pizzicannella J, Caputi S, Marchisio M, Mazzon E, Paganelli R, et al. Periodontal ligament stem cells: current knowledge and future perspectives. Stem Cells Dev 2019;28:995-1003.

18. Sharpe PT. Dental mesenchymal stem cells. Development 2016;143:2273-80.

19. Liu K, Meng H, Hou J. Activity of 25hydroxylase in human gingival fibroblasts and periodontal ligament cells. PLoS One 2012;7:e52053.

20. Diamond G, Yim S, Rigo I, McMahon L. Measuring antimicrobial peptide activity on epithelial surfaces in cell culture. Methods Mol Biol 2010;618:371-82.

21. Um S, Choi JR, Lee JH, Zhang Q, Seo B. Effect of leptin on differentiation of human dental stem cells. Oral Dis 2011;17:662-9.

22. Zhan Y, Lu R, Meng H, Wang X, Sun X, Hou J. The role of platelets in inflammatory immune responses in generalized aggressive periodontitis. J Clin Periodontol 2017;44:150-7.

23. Zhu WD, Hou JX, Liu KN, Meng HX, Tang XL. [Clinical and radiographic evaluation of class III furcation defects in the treatment using autogenous periodontal ligament cells with or without enamel matrix derivatives].[Article in Chinese]. J Peking Univ 2009;41:5661.

24. Zhu WD, Hou JX, Liu KN, Tang XL, Meng HX. [Effect of periodontal surgery on the subgingival periodontal microbiota in artificial class III furcation defects: an experimental study in monkeys].[Article in Chinese]. J Peking Univ 2012;44:22-8.

25. Li W, Zhu W, Hou J, Huang B, Liu K, 
Meng H. Leptin and its receptor expression in dental and periodontal tissues of primates. Cell Tissue Res 2014;355: 181-8.

26. Li W, Zhu W, Hou J, Meng H. Vitamin D-binding protein expression in healthy tooth and periodontium: an experimental study both in monkeys in vivo and in humans in vitro. J Periodontal Res 2017;52:755-60.

27. Ikeda $\mathrm{O}$, Egami $\mathrm{H}$, Ishiko T, Ishikawa $\mathrm{S}$, Kamohara H, Hidaka H, et al. Expression of proteinase-activated receptor-2 in human pancreatic cancer: a possible relation to cancer invasion and induction of fibrosis. Int $\mathrm{J}$ Oncol 2003;22):295-300.

28. Lan GC, Parsonage D, D'Agostino R, Torti FM, Torti SV. Regulatory effects of ferritin on angiogenesis. Proc Natl Acad Sci USA 2009;106(2):570-5.

29. Mori G, Brunetti G, Oranger A, Carbone C, Ballini A, Lo Muzio L, et al. Dental pulp stem cells: osteogenic differentiation and gene expression. Ann N Y Acad Sci 2011;1237:47-52.

30. Zarjou A, Jeney V, Arosio P, Poli M, Zavaczki E, Balla G, et al. Ferritin fer- roxidase activity: A potent inhibitor of osteogenesis. J Bone Min Res 2010;25:164-72.

31. Becs G, Zarjou A, Agarwal A, Kovács KÉ, Becs Á, Nyitrai $M$, et al. Pharmacological induction of ferritin prevents osteoblastic transformation of smooth muscle cells. J Cell Mol Med 2016;20:217-30.

32. Zarjou A, Jeney V, Arosio P, Poli M, Antal-Szalmás $\mathrm{P}$, Agarwal A, et al. Ferritin prevents calcification and osteoblastic differentiation of vascular smooth muscle cells. J Am Soc Nephrol 2009;20:1254-63.

33. Hann HW, Levy HM, Evans AE. Serum ferritin as a guide to therapy in neuroblastoma. Cancer Res 1980;40:1411-3.

34. Gray CP, Arosio P, Hersey P. Association of increased levels of heavy-chain ferritin with increased CD4+ CD25+ regulatory T-cell levels in patients with melanoma. Clin Cancer Res 2003;9:2551-9.

35. Kukulj S, Jaganjac M, Boranic M, Krizanac S, Santic Z, Poljak-Blazi M. Altered iron metabolism, inflammation, transferrin receptors, and ferritin expression in non-small-cell lung cancer. Med Oncol 2010;27:268-77.

36. Lukina EA, Levina AA, Mokeeva RA, Tokarev Yu N. The diagnostic significance of serum ferritin indices in patients with malignant and reactive histiocytosis. Br J Haematol 1993;83: 326-9.

37. Fargion S, Fracanzani A, Brando B, Arosio P, Levi S, Fiorelli G. Specific binding sites for $\mathrm{H}$-ferritin on human lymphocytes: modulation during cellular proliferation and potential implication in cell growth control. Blood 1991;78:1056-61.

38. Matzner Y, Hershko C, Polliack A, Konijn AM, Izak G. Suppressive effect of ferritin on in vitro lymphocyte function. Br J Haematol 1979;42:345-53.

39. Coffman LG, Parsonage D, D'Agostino R Jr., Torti FM, Torti SV. Regulatory effects of ferritin on angiogenesis. Proc Natl Acad Sci USA 2009;106:570-5.

40. Schonberg DL, Miller TE, Wu Q, Flavahan WA, Das NK, Hale JS, et al. Preferential iron trafficking characterizes glioblastoma stem-like cells. Cancer Cell 2015;28:441-55. 